\title{
Relative lumbar lordosis and lordosis distribution index: individualized pelvic incidence-based proportional parameters that quantify lumbar lordosis more precisely than the concept of pelvic incidence minus lumbar lordosis
}

\author{
Caglar Yilgor, MD, ${ }^{1}$ Nuray Sogunmez, MSc, ${ }^{2}$ Yasemin Yavuz, PhD, ${ }^{3}$ Kadir Abul, MD, ${ }^{2}$ \\ Louis Boissiére, MD, ${ }^{4}$ Sleiman Haddad, MD, ${ }^{5}$ Ibrahim Obeid, MD, ${ }^{4}$ Frank Kleinstück, MD, ${ }^{6}$ \\ Francisco Javier Sánchez Pérez-Grueso, MD, ${ }^{7}$ Emre Acaroğlu, MD, ${ }^{8}$ Anne F. Mannion, PhD, ${ }^{9}$ \\ Ferran Pellise, MD, ${ }^{5}$ Ahmet Alanay, MD, ${ }^{1}$ and the European Spine Study Group
}

\begin{abstract}
1Department of Orthopedics and Traumatology, Acibadem Mehmet Ali Aydinlar University School of Medicine; ${ }^{2}$ Comprehensive Spine Center, Acibadem Maslak Hospital, Istanbul; ${ }^{3}$ Department of Biostatistics, Ankara University; ${ }^{8}$ Ankara ARTES Spine Center, Ankara, Turkey; ${ }^{4}$ Spine Surgery Unit, Bordeaux University Hospital, Bordeaux, France; ${ }^{5}$ Spine Surgery Unit, Hospital Universitari Vall d'Hebron, Barcelona; ' $S p i n e$ Surgery Unit, Hospital Universitario La Paz, Madrid, Spain; Spine Center Division, Departments of ${ }^{6}$ Orthopedics and Neurosurgery and ${ }^{9}$ Research and Development, Schulthess Klinik, Zurich, Switzerland
\end{abstract}

OBJECTIVE The subtraction of lumbar lordosis (LL) from the pelvic incidence (PI) offers an estimate of the LL required for a given PI value. Relative LL (RLL) and the lordosis distribution index (LDI) are PI-based individualized measures. RLL quantifies the magnitude of lordosis relative to the ideal lordosis as defined by the magnitude of PI. LDI defines the magnitude of lower arc lordosis in proportion to total lordosis. The aim of this study was to compare RLL and PI - LL for their ability to predict postoperative complications and their correlations with health-related quality of life (HRQOL) scores.

METHODS Inclusion criteria were $\geq 4$ levels of fusion and $\geq 2$ years of follow-up. Mechanical complications were proximal junctional kyphosis/proximal junctional failure, distal junctional kyphosis/distal junctional failure, rod breakage, and implant-related complications. Correlations between PI - LL, RLL, PI, and HRQOL were analyzed using the Pearson correlation coefficient. Mechanical complication rates in PI - LL, RLL, LDI, RLL, and LDI interpreted together, and RLL subgroups for each PI - LL category were compared using chi-square tests and the exact test. Predictive models for mechanical complications with RLL and PI - LL were analyzed using binomial logistic regressions.

RESULTS Two hundred twenty-two patients (168 women, 54 men) were included. The mean age was $52.2 \pm 19.3$ years (range 18-84 years). The mean follow-up was $28.8 \pm 8.2$ months (range 24-62 months). There was a significant correlation between $\mathrm{PI}-\mathrm{LL}$ and $\mathrm{PI}(r=0.441, p<0.001)$, threatening the use of $\mathrm{PI}-\mathrm{LL}$ to quantify spinopelvic mismatch for different PI values. RLL was not correlated with PI $(r=-0.093, p>0.05)$; therefore, it was able to quantify divergence from ideal lordosis for all PI values. Compared with PI - LL, RLL had stronger correlations with HRQOL scores $(p<$ 0.05). Discrimination performance was better for the model with RLL than for PI - LL. The agreement between RLL and $P I-L L$ was high $(\kappa=0.943, p<0.001)$, moderate $(\kappa=0.455, p<0.001)$, and poor $(\kappa=-0.154, p=0.343)$, respectively, for large, average, and small PI sizes. When analyzed by RLL, each PI - LL category was further divided into distinct groups of patients who had different mechanical complication rates $(p<0.001)$.

CONCLUSIONS Using the formula of PI - LL may be insufficient to quantify normolordosis for the whole spectrum of $\mathrm{PI}$ values when applied as an absolute numeric value in conjunction with previously reported population-based average thresholds of $10^{\circ}$ and $20^{\circ}$. Schwab PI - LL groups were found to constitute an inhomogeneous group of patients. RLL offers an individualized quantification of LL for all PI sizes. Compared with PI - LL, RLL showed a greater association

ABBREVIATIONS AUC = area under the receiver operating characteristic curve; COMI = Core Outcomes Measures Index; GAP = global alignment and proportion; $\mathrm{HRQOL}=$ health-related quality of life; LDI = lordosis distribution index; LIV = lowest instrumented vertebra; LL = lumbar lordosis; NPV = negative predictive value; ODI = Oswestry Disability Index; PAC = percentage accuracy in classification; $\mathrm{PI}=$ pelvic incidence; $\mathrm{PPV}=$ positive predictive value; $\mathrm{PT}=$ pelvic tilt; $\mathrm{RLL}=$ relative $\mathrm{LL} ; \mathrm{SF}-36$ = 36-Item Short-Form Health Survey; SRS = Scoliosis Research Society; UIV = upper instrumented vertebra.

SUBMITTED July 31, 2017. ACCEPTED August 4, 2017.

INCLUDE WHEN CITING DOI: 10.3171/2017.8.FOCUS17498. 
with both mechanical complications and HRQOL. The use of RLL and LDI together, instead of PI - LL, for surgical planning may result in lower mechanical complication rates and better long-term HRQOL.

https://thejns.org/doi/abs/10.3171/2017.8.FOCUS17498

KEY WORDS adult spinal deformity; mechanical complication; spinopelvic mismatch; individualized analysis; compensatory mechanisms; distribution of lordosis

$\mathrm{T}$ o allow the human trunk to adopt an erect posture, the spine has developed successive, opposing curves, which differ from the C-shaped spine of bipedal primates. No other species has spinal alignment that includes a lumbar lordosis (LL). ${ }^{4,29}$ This upright bipedalism provided notable modifications in the morphology of the skeleton. ${ }^{13}$ Sitting upon the femoral heads, the pelvis has rotated vertically to act as the foundation for the vertebral column. ${ }^{14}$

Reported normal values for LL in asymptomatic adults vary widely. ${ }^{5,9,19,30,31}$ After the description of pelvic incidence (PI), sacral slope, and pelvic tilt (PT) to evaluate the size and orientation of the pelvis, ${ }^{7}$ it is now understood that the magnitude of ideal lordosis is specific to the given individual and should be estimated based on the PI. Depending on the value of PI, any given Cobb value can be hypolordotic, normal lordotic, or hyperlordotic. The formula of PI minus LL (PI - LL) offers an estimate of the lordosis required for a given PI value and tries to quantify the mismatch between pelvic morphology and the lumbar curve. Schwab et al. suggested that a PI $-\mathrm{LL}<10^{\circ}$ represents satisfactory spinopelvic alignment and incorporated this into an adult spinal deformity classification. ${ }^{24-26}$

Although it is a useful predictor, this simple approach has limitations. PI - LL is easy to calculate, yet hard to evaluate, because it also needs to be adapted to the pelvic morphology of each patient. Patients with so-called small PI have been reported to require an LL in excess of their PI $\left(\mathrm{LL}=\mathrm{PI}+10^{\circ}\right)$, whereas patients with large PI require an LL smaller than their PI $\left(\mathrm{LL}=\mathrm{PI}-10^{\circ}\right) .{ }^{27}$ However, it is not clear where the thresholds for small and large PI should lie.

Therefore, the concept of using PI - LL solely as an absolute numeric value with population-based average thresholds may be misleading. Arbitrary use of the +9 or -9 further complicates the reproducibility of preoperative planning.

Relative LL (RLL) and the lordosis distribution index (LDI) are 2 of 4 PI-based proportional radiographic parameters that comprise the global alignment and proportion (GAP) score ${ }^{34}$ RLL quantifies the magnitude of lordosis relative to the ideal lordosis as defined by the magnitude of PI. RLL is described as the ideal LL subtracted from the measured LL, assessing the magnitude of hypoand hyperlordosis as divergence from the calculated ideal.

However, even the magnitude of lordosis as quantified by a single Cobb value is insufficient to fully describe the lordotic curve. Through a horizontal line crossing the apex of the curve, lordosis can be reconstructed into 2 tangent arcs of a circle. ${ }^{6}$ This allows a better understanding of the distribution of lordosis as upper- and lower-arc lordosis. The LDI defines the magnitude of lower-arc lordosis relative to the total lordosis. ${ }^{34}$ The LDI is described as L4-S1 lordosis/L1-S1 lordosis $\times 100$.
The hypothesis of the present study was that the Schwab PI - LL categories would comprise an inhomogeneous group of patients with different mechanical complication rates, indicating that PI - LL is insufficient to quantify spinopelvic mismatch. The aim of this study was to compare RLL and PI - LL for their ability to predict mechanical complications and their correlations with health-related quality of life (HRQOL) scores.

\section{Methods}

\section{Patient Inclusion Criteria}

This was a retrospective analysis of data collected in the multicenter, consecutive, prospective study of the European Spine Study Group, a collaboration of spine surgeons from 6 sites. Institutional review board approval was obtained at each site. Enrollment criteria were patient age $\geq 18$ years with at least 1 of the following: coronal Cobb angle $\geq 20^{\circ}$, sagittal vertical axis $\geq 5 \mathrm{~cm}, \mathrm{PT} \geq 25^{\circ}$, or thoracic kyphosis $\geq 60^{\circ}$. For the present study, the specific inclusion criteria were $\geq 4$ levels of posterior instrumented fusion and $\geq 2$ years of follow-up.

\section{Outcome Data}

Mechanical complications were defined as proximal junctional kyphosis and proximal junctional failure; distal junctional kyphosis and distal junctional failure; rod breakage and other implant-related complications (not related to proximal junctional failure), such as screw loosening/ breakage/pullout; or interbody graft, hook, or set-screw dislodgements. Proximal junctional failure was defined as $\geq 10^{\circ}$ increase in kyphosis between the upper instrumented vertebra (UIV) and the UIV +2 between early postoperative and follow-up radiographs. Proximal junctional failure was defined as fracture of the UIV or UIV + 1, pullout of instrumentation at the UIV, and/or presence of sagittal subluxation. Distal junctional kyphosis/distal junctional failure was defined as $\geq 10^{\circ}$ postoperative increase in kyphosis between the lowest instrumented vertebra (LIV) and LIV -1 and/or pullout of instrumentation at the LIV.

\section{Radiographic Evaluation}

PI, L4-S1 lordosis, and L1-S1 lordosis were measured on radiographs obtained 6 weeks postoperatively using a validated software (Surgimap; Nemaris, Inc.). PI - LL was calculated using the following formula: PI minus L1-S1 lordosis. RLL was calculated using the following formula: L1-S1 lordosis minus $(0.62 \times \mathrm{PI}+29)$. The LDI was calculated using the following formula: L4-S1 lordosis/L1-S1 lordosis $\times 100$.

PI - LL was divided into 3 categories as $0,+$, and ++ for PI - LL within $10^{\circ}$, between $10^{\circ}$ and $20^{\circ}$, and $>20^{\circ}$, respectively. RLL and LDI were divided into 4 subgroups. 
RLL $<-25^{\circ}$ was considered severe hypolordosis; $-25^{\circ}$ to $-14.1^{\circ}$, moderate hypolordosis; $-14^{\circ}$ to $11^{\circ}$, aligned; and $>$ $11^{\circ}$, hyperlordosis. An LDI $<40 \%$ was considered severe hypolordotic maldistribution; $40 \%-49 \%$, moderate hypolordotic maldistribution; $50 \%-80 \%$, aligned; and $>80 \%$, hyperlordotic maldistribution. ${ }^{34}$

\section{HRQOL Measures}

The following questionnaires, which were completed preoperatively and at the last follow-up, were used in the analyses: Oswestry Disability Index (ODI.v2), ${ }^{8}$ Core Outcomes Measures Index (COMI), ${ }^{16}$ Scoliosis Research Society (SRS)-22 questionnaire, ${ }^{1}$ and 36-Item Short-Form Health Survey (SF-36). ${ }^{33}$

\section{Statistical Analysis}

Preliminary analyses showed the relationship to be linear, with all variables normally distributed, as assessed by Shapiro-Wilk's test $(p>0.05)$, and there were no outliers. Two separate Pearson's product-moment correlations were run to assess the relationships between 1) RLL and PI, and 2) PI - LL and PI. One-way ANOVA and post hoc Tukey honest significant difference tests were used to compare PI values in RLL subgroups and PI - LL categories.

A Pearson's partial correlation was run to determine the relationship between RLL, PI - LL, and final followup HRQOL scores (controlling for preoperative HRQOL scores). Absolute RLL and PI - LL values were used in the analysis.

Two separate binomial logistic regressions were performed to ascertain the effects of RLL and PI - LL on the likelihood of incurring a postoperative mechanical complication. Overall, performance of the models was assessed using Nagelkerke $\mathrm{R}^{2} \cdot{ }^{17}$ As discrimination measures, the area under the receiver operating characteristic curve (AUC), percentage accuracy in classification (PAC), sensitivity, specificity, positive predictive value (PPV), and negative predictive value (NPV) were calculated.

The agreement of RLL and PI - LL for average, small, and large values of PI was assessed using $\kappa$ statistics.

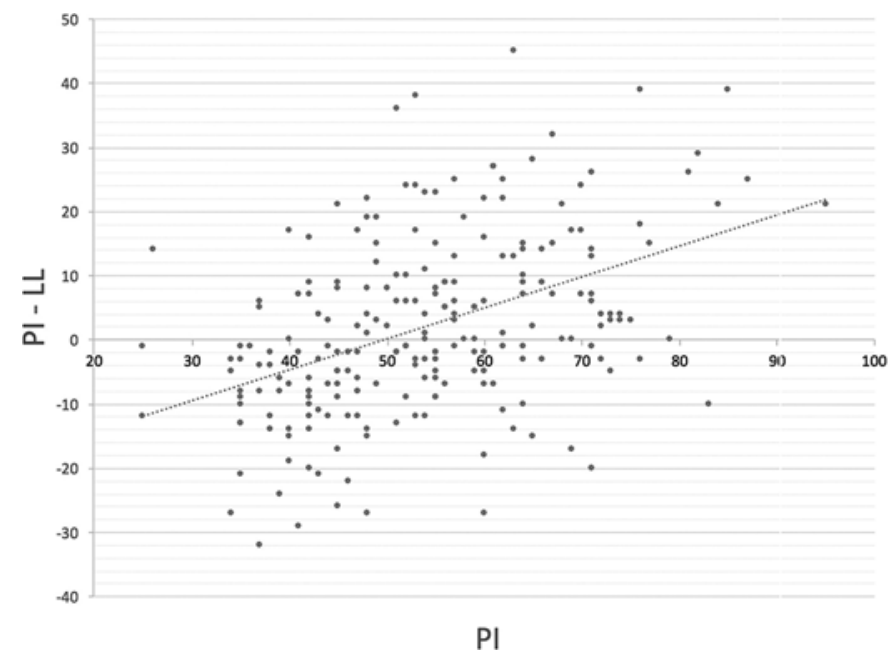

Mechanical complication rates in RLL subgroups for each of the PI - LL categories were compared using chisquare tests and the exact test.

To account for both the magnitude and distribution of lordosis, the RLL and LDI categories were combined to form 4 additional categories as follows: 1) RLL and LDI aligned, 2) RLL or LDI moderately malaligned, 3) RLL and LDI moderately malaligned, and 4) RLL and/or LDI severely malaligned. Mechanical complication rates in the categories for PI - LL, RLL, LDI, and RLL and LDI interpreted together were compared using chi-square tests.

\section{Results}

\section{Patient Clinical Characteristics}

The European Spine Study Group database contained 631 operative patients, 548 of whom had $\geq 4$ levels of posterior fusion. Among these patients, 251 were $\geq 2$ years postoperative; 29 patients (11.6\%) were lost to follow-up, which left 222 patients to be included.

The mean age of patients was $52.2 \pm 19.3$ years (range $18-84$ years), and the mean duration of follow-up was 28.8 \pm 8.2 months (range $24-62$ months).

\section{Mechanical Complications}

One hundred patients (45\%) experienced at least 1 type of mechanical complication. Twenty patients $(9 \%)$ had proximal junctional kyphosis, 41 (18.5\%) had proximal junctional failure, 42 (18.9\%) had rod breakage, 14 (6.3\%) had implant-related complications, and 5 (2.3\%) had distal junctional kyphosis. Fifty patients $(22.5 \%)$ had undergone at least 1 revision surgery due to mechanical failure. Twenty patients $(9 \%)$ and 9 patients $(4.1 \%)$ had undergone 2 and $\geq 3$ such revisions, respectively.

\section{Dependence of RLL and PI - LL on PI}

There was a significant correlation between PI - LL and PI $(r=0.441, p<0.001)$, threatening the use of PI $-\mathrm{LL}$ to quantify spinopelvic mismatch over a range of PI values (Fig. 1). RLL was not correlated with PI $(r=-0.093$, $p>$

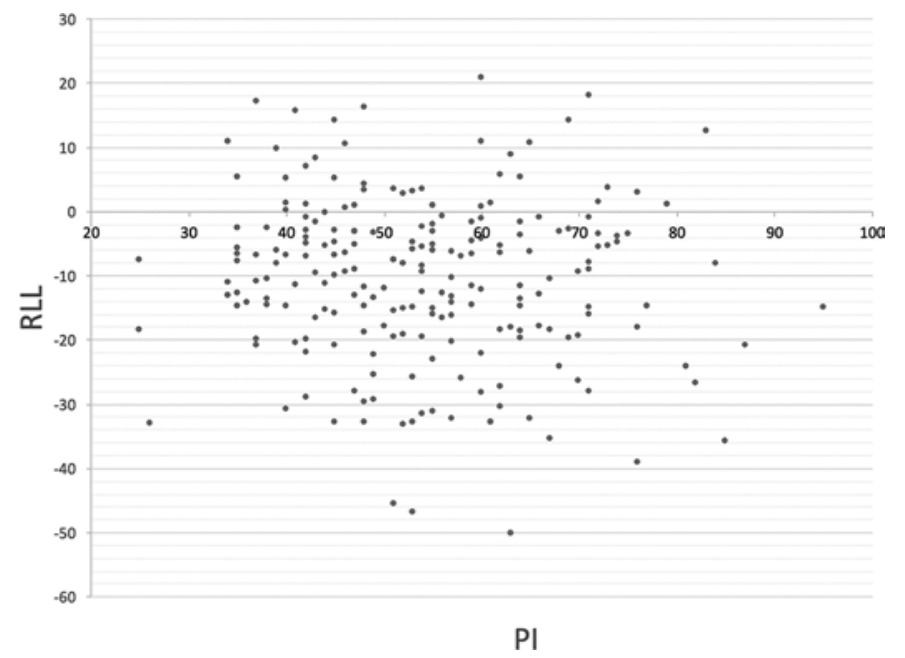

FIG. 1. Correlations between PI and preoperative PI - LL (left) and RLL (right) values. 
TABLE 1. PI values among PI - LL and RLL categories

\begin{tabular}{|c|c|c|c|c|}
\hline Category & $\begin{array}{c}\text { No. of } \\
\text { Patients }\end{array}$ & $\begin{array}{l}\text { Mean PI } \\
\pm S D\left({ }^{\circ}\right)\end{array}$ & $\begin{array}{c}\text { Min to } \\
\operatorname{Max} \mathrm{PI}\left(\left(^{\circ}\right)\right.\end{array}$ & $\begin{array}{c}p \\
\text { Value }\end{array}$ \\
\hline$P I-L L$ & & & & $<0.05$ \\
\hline 0 & 124 & $53 \pm 11.5$ & $25-79$ & \\
\hline+ & 60 & $53.1 \pm 13$ & $25-83$ & \\
\hline++ & 38 & $59.4 \pm 15.5$ & $34-95$ & \\
\hline RLL & & & & $>0.05$ \\
\hline Aligned & 131 & $52.6 \pm 12.1$ & $25-84$ & \\
\hline Hyperlordosis & 8 & $56.8 \pm 16.5$ & $37-83$ & \\
\hline Moderate hypolordosis & 51 & $55.7 \pm 14.2$ & $25-95$ & \\
\hline Severe hypolordosis & 32 & $57.1 \pm 12.1$ & $26-85$ & \\
\hline
\end{tabular}

$\mathrm{SD}=$ standard deviation.

0.05); therefore, it was able to quantify divergence from ideal lordosis for all PI values (Fig. 1).

Postoperatively, in reference to PI - LL thresholds of Schwab's classification, 124 patients $(55.9 \%)$ fell into the 0 category, whereas $60(27 \%)$ and $38(17.1 \%)$ fell into the + and ++ categories, respectively. Patients in the ++ category were found to have larger mean PI values than those in the categories of 0 and $+(\mathrm{F}(2,219)=4.027, \mathrm{p}=0.019$, $\mathrm{p}<0.05)$ (Table 1). Thus, PI - LL categories comprised patients with different PI values.

Postoperatively, in reference to RLL, 131 patients (59\%) were aligned, whereas 8 (3.6\%), 51 (23\%), and 32 (14.4\%) were hyperlordotic, moderately hypolordotic, and severely hypolordotic, respectively. Mean PI values were not significantly different in RLL subgroups $(F(3,218)=$ $1.563, \mathrm{p}=0.199, \mathrm{p}>0.05$ ) (Table 1). Thus, in contrast to categorization with PI - LL, RLL subgroups comprised patients with different lordotic states, not patients with different PI values.

\section{Correlations to HRQOL Scores}

Compared with PI - LL, RLL had stronger partial correlations with ODI, COMI, SF-36 Physical Component Summary and SF-36 Mental Component Summary, and

TABLE 2. Partial correlations between RLL, PI - LL, and HRQOL scores

\begin{tabular}{lcccccc}
\hline & \multicolumn{2}{c}{$\mathrm{PI}-\mathrm{LL}$} & & \multicolumn{2}{c}{$\mathrm{RLL}$} \\
\cline { 2 - 3 } \cline { 6 - 7 } \multicolumn{1}{c}{ HRQOL Scores } & $\mathrm{r}$ & $\mathrm{p}$ Value & & $\mathrm{r}$ & $\mathrm{p}$ Value \\
\hline ODI & 0.203 & 0.004 & & 0.283 & 0.000 \\
\hline COMI & 0.179 & 0.040 & & 0.217 & 0.012 \\
\hline SRS-22 subtotal & -0.136 & 0.052 & & -0.232 & 0.001 \\
\hline SRS-22 function & -0.115 & 0.102 & & -0.220 & 0.002 \\
\hline SRS-22 pain & -0.185 & 0.008 & & -0.206 & 0.003 \\
\hline SRS-22 mental health & -0.069 & 0.330 & & -0.126 & 0.072 \\
\hline SRS-22 self image & -0.050 & 0.478 & & -0.163 & 0.020 \\
\hline SF-36 PCS & -0.108 & 0.129 & & -0.168 & 0.018 \\
\hline SF-36 MCS & -0.125 & 0.079 & & -0.144 & 0.043 \\
\hline
\end{tabular}

MCS = Mental Component Summary; PCS = Physical Component Summary.
TABLE 3. Univariate logistic regression analysis: prediction of mechanical complications with RLL and PI - LL

\begin{tabular}{lcrlr}
\hline \multicolumn{1}{c}{ Variable } & MC $\%$ & OR & $95 \% \mathrm{Cl}$ & $\mathrm{p} \mathrm{Value}$ \\
\hline $\mathrm{PI}-\mathrm{LL}$ category & & & & \\
\hline 0 & 37.9 & 1.0 & & \\
\hline+ & 40 & 1.1 & $0.6-2.1$ & 0.784 \\
\hline++ & 76.3 & 5.3 & $2.3-12.1$ & $<0.001$ \\
\hline RLL subgroup & & & & \\
\hline Aligned & 23.7 & 1.0 & & \\
\hline Hyperlordosis & 87.5 & 22.6 & $2.7-190.7$ & 0.004 \\
\hline$\quad$ Moderate hypolordosis & 64.7 & 5.9 & $2.9-11.9$ & 0.000 \\
\hline$\quad$ Severe hypolordosis & 90.6 & 31.2 & $8.9-109.4$ & 0.000 \\
\hline
\end{tabular}

$\mathrm{Cl}=$ confidence interval; $\mathrm{MC}=$ mechanical complications; $\mathrm{OR}=$ odds ratio.

all SRS-22 subdomain scores at last follow-up, controlling for preoperative scores (Table 2).

\section{Prediction of Mechanical Complications With RLL and PI - LL}

Higher PI - LL was associated with an increased likelihood of mechanical complications. Patients who had a PI - LL of $10^{\circ}-20^{\circ}$ and $>20^{\circ}$ had 1.1 and 5.3 times greater odds of experiencing a mechanical complication, respectively, than patients who had a PI $-\mathrm{LL}<10^{\circ}$ (Table 3).

The model for PI - LL explained 10.8\% (Nagelkerke $\mathrm{R}^{2}$ ) of the variance in incurring a mechanical complication, with $29 \%$ sensitivity, $92.6 \%$ specificity, $76.3 \%$ PPV, 61.4\% NPV, 0.62 AUC, and 64\% PAC (Table 4).

Worse RLL, in both hyperlordotic and hypolordotic directions, was associated with an increased likelihood of incurring a mechanical complication. Hyperlordotic patients had 22.6 times greater odds of experiencing a mechanical complication than aligned patients. Moderately and severely hypolordotic patients had 5.9 and 31.2 times greater odds of experiencing a mechanical complication than aligned patients (Table 3 ).

The model for RLL explained 36.2\% (Nagelkerke $\mathrm{R}^{2}$ ) of the variance in incurring a mechanical complication and correctly classified $76.1 \%$ of cases with $69 \%$ sensitivity, $82 \%$ specificity, $75.8 \%$ PPV, $76.3 \%$ NPV, and 0.78 AUC (Table 4).

TABLE 4. Univariate logistic regression analysis: performance of the models for PI - LL and RLL

\begin{tabular}{lcc}
\hline \multicolumn{1}{c}{ Variable } & $\mathrm{PI}-\mathrm{LL}$ & $\mathrm{RLL}$ \\
\hline Significance of the model (p value) & 0.000 & 0.000 \\
\hline${\text { Nagelkerke } \mathrm{R}^{2}}$ & 0.108 & 0.362 \\
\hline AUC (SE) & $0.615(0.003)$ & $0.776(0.033)$ \\
\hline PAC, $\%$ & 64 & 76.1 \\
\hline Sensitivity, \% & 29 & 69 \\
\hline Specificity, $\%$ & 92.6 & 82 \\
\hline PPV, $\%$ & 76.3 & 75.8 \\
\hline NPV, $\%$ & 61.4 & 76.3 \\
\hline
\end{tabular}

$\mathrm{SE}=$ standard error. 
TABLE 5. Agreement of RLL and PI - LL in patients with small, average, and large PI

\begin{tabular}{|c|c|c|c|c|c|c|}
\hline \multirow[b]{2}{*}{ PI - LL category } & \multicolumn{2}{|c|}{ Small PI, $<41^{\circ}$} & \multicolumn{2}{|c|}{ Average $\mathrm{PI}, 41^{\circ}-67^{\circ}$} & \multicolumn{2}{|c|}{ Large $\mathrm{PI},>67^{\circ}$} \\
\hline & 0 & $+/++$ & 0 & $+/++$ & 0 & +/++ \\
\hline \multicolumn{7}{|l|}{ RLL subgroup, no. of patients } \\
\hline Aligned & 12 & 12 & 68 & 21 & 17 & 1 \\
\hline Moderate hypolordosis/severe hypolordosis/hyperlordosis & 8 & 4 & 19 & 43 & 0 & 17 \\
\hline к statistics (SE) & \multicolumn{2}{|c|}{$-0.154(0.158)$} & \multicolumn{2}{|c|}{$0.455(0.073)$} & \multicolumn{2}{|c|}{$0.943(0.056)$} \\
\hline $\mathrm{p}$ value & \multicolumn{2}{|c|}{0.343} & \multicolumn{2}{|c|}{$<0.001$} & \multicolumn{2}{|c|}{$<0.001$} \\
\hline
\end{tabular}

In terms of performance measures, the model for RLL was superior to that for PI - LL (Table 4), although both of the models were statistically significant $(\mathrm{p}<0.001)$.

\section{PI-Based Individualization of the Quantification of Spinopelvic Mismatch}

Because the magnitude of the PI - LL is affected by the magnitude of PI, using PI - LL solely as an absolute numeric value may be misleading. The mean PI value in this study was $54^{\circ} \pm 13^{\circ}$ (minimum 25 , maximum 95 ), showing a normal distribution (Kolmogorov-Smirnov test, $\mathrm{p}=0.043$ ). The agreement of classification based on PI LL and RLL was calculated for 3 groups: PI mean \pm 1 SD (average PI), PI < mean - $1 \mathrm{SD}$ (small PI), and PI > mean +1 SD (large PI) (Table 5).

In patients with average PI (which corresponds to $68 \%$ of the population), the agreement between RLL and PI LL was moderate $(\kappa=0.455, \mathrm{p}<0.001)$. The agreement was high for patients with large PI $(\kappa=0.943, \mathrm{p}<0.001)$, and the agreement was poor and no better than chance for those with small PI $(\kappa=-0.154, \mathrm{p}=0.343)$.

In patients with average PI, the mechanical complication rates were $25 \%$ and $76.7 \%$, respectively, for those who were aligned $(n=68)$ and malaligned $(n=43)$, according to both RLL and PI - LL. For the 21 patients for whom PI - LL was + or ++, yet RLL was aligned, the mechanical complication rate was $9.5 \%$. On the contrary, for the 19 patients for whom PI - LL was 0, yet RLL detected malalignment, the mechanical complication rate was $63.1 \%$. These findings indicate that PI - LL can be misleading even in patients with average PI values.

\section{PI - LL Categories Analyzed by RLL}

To compare categorization of spinopelvic mismatch using the PI - LL concept with that of the PI-based individualized parameter of RLL, postoperative PI - LL categories were analyzed in relation to RLL category. Cross- tabulation of the quantification of the lordosis by RLL and PI - LL is shown in Table 6.

One hundred twenty-four patients were postoperatively classified as PI - LL 0. When these patients were analyzed by RLL, 97 (78.2\%) were aligned and 27 (21.8\%) were moderately hypolordotic. These 2 distinct groups of patients were found to have significantly different mechanical complication rates $(\mathrm{p}<0.001)$ (Fig. 2). While the mean PI value was $54.8^{\circ} \pm 11.5^{\circ}$ in RLL-aligned patients, the mean PI value was $46.2^{\circ} \pm 8.5^{\circ}$ in RLL-moderately hypolordotic patients. The PI - LL cutoff of within $10^{\circ}$ was ineffective as a criterion for determining whether there was any spinopelvic mismatch in these patients with relatively small PI.

Sixty patients were postoperatively classified as PI LL +. When these patients were analyzed by RLL, 28 $(46.7 \%)$ were aligned, whereas $3(5 \%)$ were hyperlordotic, $20(33.3 \%)$ were moderately hypolordotic, and $9(15 \%)$ were severely hypolordotic. Mean PI values were $46^{\circ} \pm$ $10^{\circ}, 74.3^{\circ} \pm 7.6^{\circ}, 63.1^{\circ} \pm 8.1^{\circ}$, and $45.8^{\circ} \pm 9.1^{\circ}$, respectively. Thus, although they were all classified as PI - LL + , these groups of patients had different magnitudes of PI. These 4 distinct groups of patients were also found to have significantly different mechanical complication rates $(\mathrm{p}=0.000)$ (Fig. 2).

Thirty-eight patients were postoperatively classified as PI - LL ++. When these 38 patients were analyzed by RLL, 6 (15.8\%) were aligned, whereas 5 (13.2\%) were hyperlordotic, $4(10.5 \%)$ were moderately hypolordotic, and $23(60.5 \%)$ were severely hypolordotic. Mean PI values were $46.8^{\circ} \pm 18.8^{\circ}, 46.2^{\circ} \pm 8.8^{\circ}, 82.8^{\circ} \pm 11.4^{\circ}$, and $61.5^{\circ}$ $\pm 10.2^{\circ}$, respectively. These 4 distinct groups of patients were also found to have significantly different mechanical complication rates $(\mathrm{p}=0.001)$ (Fig. 2). Thus, patients with different PI sizes, who would otherwise have been collectively considered as PI $-\mathrm{LL}++$, displayed different lordotic states according to RLL, in whom mechanical complication rates were significantly different.

TABLE 6. Cross-tabulation of PI - LL by RLL

\begin{tabular}{cccccc}
\hline PI - LL & \multicolumn{5}{c}{ RLL Group, No. of Patients (\%) } \\
\cline { 2 - 6 } Category & Hyperlordosis & Aligned & Moderate Hypolordosis & Severe Hypolordosis & Total \\
\hline 0 & $0(0)$ & $97(78.2)$ & $27(21.8)$ & $0(0)$ & $124(100)$ \\
\hline+ & $3(5)$ & $28(46.7)$ & $20(33.3)$ & $9(15)$ & $60(100)$ \\
\hline++ & $5(13.2)$ & $6(15.8)$ & $4(10.5)$ & $23(60.5)$ & $38(100)$ \\
\hline Total & $8(3.6)$ & $131(59)$ & $51(23)$ & $32(14.4)$ & $222(100)$ \\
\hline
\end{tabular}




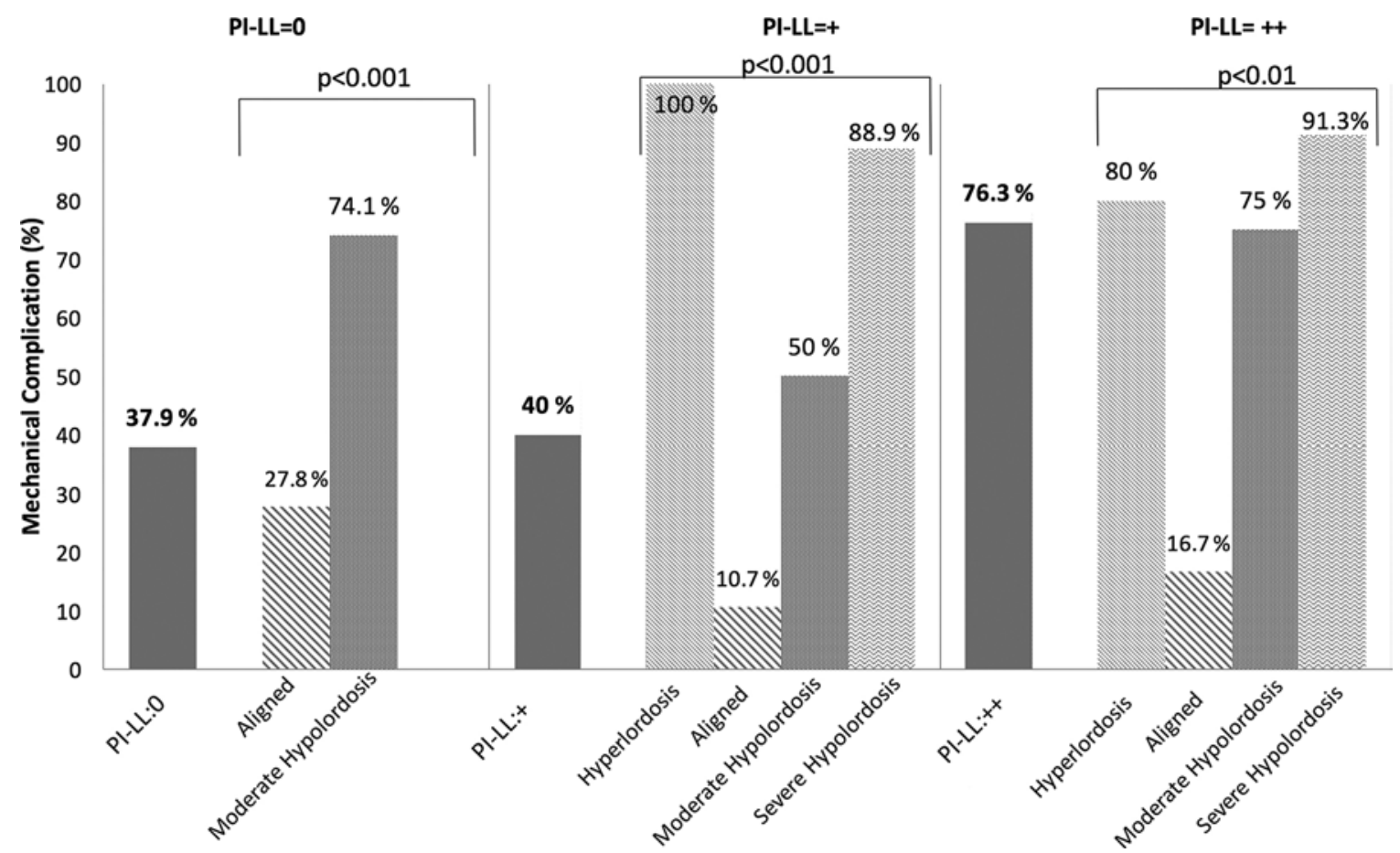

FIG. 2. Percentage of mechanical complications among RLL subgroups in each postoperative PI - LL category.

\section{Mechanical Complication Rates According to RLL and LDI Interpreted Together}

The distribution of the lordosis in the lower (L4-S1) and upper (L1-3) arcs is important because it influences the distribution of loads. ${ }^{22}$ Thus, a maldistribution of lordosis may cause mechanical complications, which is not considered in the Schwab criteria.

Without considering the magnitude of lordosis, when the distribution of lordosis postoperatively was analyzed with the LDI, $147(66.2 \%)$ patients were postoperatively aligned, whereas 41 (18.5\%), 23 (10.4\%), and 11 (5\%) patients had hyperlordotic maldistribution, moderately hypolordotic maldistribution, and severely hypolordotic maldistribution of lordosis, respectively. These patients were found to have significantly different mechanical complication rates of $27.2 \%, 87.8 \%, 69.6 \%$, and $72.7 \%$, respectively $\left(\chi^{2}=58.1626[\mathrm{DF}=3], \mathrm{p}<0.001\right)$.

Lordosis, however, should be described using both distribution and magnitude parameters. RLL and LDI both being aligned describes the state where the lordosis is restored to the ideal magnitude and distribution. The percentage of mechanical complications significantly increased as the RLL and LDI subgroups worsened (Table 7).

\section{Discussion}

SRS-Schwab sagittal parameters set surgical goals as PI - LL within $10^{\circ}$, sagittal vertical axis $<4 \mathrm{~cm}$, and PT $<20^{\circ}$ for achieving good HRQOL. ${ }^{25}$ However, the use of PI - LL may occasionally be misleading because it is oversimplistic and it uses an absolute numeric value with population-based average thresholds. Moreover, lumbar lordosis, which does not exist in any species other than humans, cannot be adequately defined and quantified by a single Cobb angle value.

The PI-based proportional parameter of RLL provides a more precise and individualized interpretation of the lordosis. The fact that the PI - LL and PI were correlated in this study indicates that PI - LL has a reduced ability to quantify spinopelvic harmony for different PI values. A higher or lower value of PI - LL may be due to 2 entities: the size of the pelvis and the magnitude of lordosis. On the other hand, RLL and PI were not correlated; that is, an increase in PI was not associated with an increase or a decrease in RLL. Therefore, a higher or lower value of RLL directly represents the magnitude of lordosis in terms of the deviation from the ideal.

Simplified thresholds may be easy to remember and useful, but they increase the margin of error. The concept of PI - LL provides a pragmatic method of analysis. Nevertheless, with the evolving understanding in medicine and the ready availability of computational methods, one's duty is to offer patient-specific solutions. Proportional analysis of the magnitude of the lordosis offers better categorization that is tailored to each individual. The use of RLL clearly pointed out that the so-called Schwab PI - LL 0, + , and ++ categories actually constituted inhomogeneous patient groups with differing mean PI values and mechanical complication rates. When LDI is used with RLL to account simultaneously for the magnitude and distribution of the lordosis, the distinction is even greater.

The surgical goal dictating that achievement of a PI $\mathrm{LL}<10^{\circ}$ is associated with good results is, therefore, not true for every patient. The current study indicates that the surgical target of PI $-\mathrm{LL}<10^{\circ}$ resulted in a $37.9 \%$ rate of overall mechanical complications. In contrast, when 
TABLE 7. Percentages of mechanical complications according to postoperative RLL and LDI subgroups interpreted together

\begin{tabular}{|c|c|c|c|c|}
\hline \multirow[b]{2}{*}{ Magnitude \& Shape of Lordosis } & \multicolumn{2}{|c|}{ Mechanical Complications, No. (\%) } & \multirow[b]{2}{*}{$\chi^{2}(\mathrm{DF}=3)$} & \multirow[b]{2}{*}{$\mathrm{p}$ Value } \\
\hline & No & Yes & & \\
\hline RLL \& LDI aligned & $90(87.4)$ & $13(12.6)$ & \multirow{4}{*}{93.03} & \multirow{4}{*}{$<0.001$} \\
\hline RLL or LDI moderately malaligned & $19(52.8)$ & $17(47.2)$ & & \\
\hline RLL \& LDI moderately malaligned & $2(28.6)$ & $5(71.4)$ & & \\
\hline RLL \&/or LDI severely malaligned & $12(15.8)$ & $64(84.2)$ & & \\
\hline
\end{tabular}

alignment was achieved based on the magnitude and distribution of lordosis, as quantified by RLL and LDI, the mechanical complication rate was just $12.6 \%$.

\section{RLL, LDI, and Mechanical Complications}

Postoperatively, 103 patients were RLL and LDI aligned. Thirteen of these patients (12.6\%) experienced mechanical complications. The mean GAP score of these 13 patients was 3 (range $0-7$ ), suggesting that although these patients were well aligned relative to the magnitude and distribution of lordosis, they were disproportioned either in the pelvic and/or global spinopelvic alignment. As previously reported, mechanical complication rates can be as low as $6.1 \%$ when patients are proportioned in the sagittal plane according to GAP score. ${ }^{34}$

\section{RLL and LDI in Other Normative Data Studies}

The ideal value for LL was determined from simple linear regression analyses of its relationship with PI values, using data from asymptomatic volunteers recorded in an adult radiography database. ${ }^{34}$ Ideal $\mathrm{LL}=0.62 \times \mathrm{PI}+29$ $\left(\mathrm{R}^{2}=0.39, \mathrm{p}<0.001\right)$. LL is considered to be aligned when it is between $11^{\circ}$ of hyperlordosis and $14^{\circ}$ of hypolordosis from this calculated ideal. The distribution of the lordosis was considered to be aligned if the LDI was within the range $50 \%-80 \%$.

In 1998, Legaye et al. ${ }^{15}$ evaluated 49 subjects with a mean age of 24 years and found that the mean PI was $52^{\circ}$ and the mean LL was $60^{\circ}$. RLL can thus be calculated as $1.2^{\circ}$. Similar analysis of normative data studies by Vialle et al..$^{32}$ and Roussouly et al..$^{20,21}$ revealed $3.4^{\circ}, 0.2^{\circ}$, and $0.8^{\circ}$ of normolordosis, respectively. A review by Kuntz et al. ${ }^{11}$ in 2007 that comprises 1094 asymptomatic adults revealed that the mean PI was $54^{\circ}$ and the mean LL was $62^{\circ}$, resulting in an RLL of $-0.5^{\circ}$. This indicates the universal usability of RLL.

Barrey et al. ${ }^{2}$ studied spinopelvic parameters in 154 subjects, dividing them into 6 PI groups. From their data, RLL can be calculated as $2.4^{\circ}, 0.0^{\circ},-0.1^{\circ}, 0.5^{\circ}, 0.9^{\circ}$, and $-3.5^{\circ}$ for PI groups of $28^{\circ}-37.9^{\circ}, 38^{\circ}-47.9^{\circ}, 48^{\circ}-57.9^{\circ}$, $58^{\circ}-67.9^{\circ}, 68^{\circ}-77.9^{\circ}$, and $78^{\circ}-87.9^{\circ}$, respectively (the $78^{\circ}-87.9^{\circ}$ group only had 2 patients). This finding demonstrates the ability of the individualized RLL to measure the spinopelvic mismatch for all magnitudes of PI.

Contact force distribution depends on the vertebral plate orientation within the curvature. Therefore, the distribution of the lordosis is as important as the magnitude. Roussouly and Pinheiro-Franco ${ }^{23}$ classified lordotic orientation into 3 groups: backward-tilted, well-balanced, and forward-tilted lordosis. The authors of the current study believe that such a classification will not cover the whole spectrum; hence, we propose the use of a proportional formulation instead. Lordosis should be quantified as the magnitude of lordosis with regard to one's ideal in proportion to PI (that is, RLL), plus the magnitude of lower-arc lordosis with regard to the total lordosis (that is, LDI).

The validity of LDI is supported by the current literature on normative data. Research in asymptomatic individuals has revealed that the L4-S1 segment provides approximately two-thirds of the total LL. $3,10,20$ A review of the English-language literature revealed that the reported mean L4-5 lordosis is $41^{\circ}$ and the mean L1-S1 lordosis is $68^{\circ}$, resulting in an LDI of $60 \% .{ }^{11}$ Roussouly et al. ${ }^{20}$ reported similar values for lower-arc lordosis and total lordosis of $39.92^{\circ}$ and $61.42^{\circ}$, respectively, resulting in an LDI of $64.9 \%$.

\section{RLL and LDI in Patients With Adult Spinal Deformity}

Today, it is crystal clear that aging and degeneration is a kyphosing process in which LL diminishes and thoracic kyphosis increases. In a study of 125 patients with adult spinal deformity reported by Lafage et al., ${ }^{12}$ RLL was calculated as $-19.1^{\circ}$, displaying moderate hypolordosis on average. Obeid et al. ${ }^{18}$ studied patients with more severe deformity, with a mean knee flexion angle of $14.3^{\circ}$. RLL was calculated as $-41.4^{\circ}$, indicating severe hypolordosis.

The compensatory mechanisms, which act voluntarily or involuntarily, alter the magnitude of the curves as well as their shape. In the setting of lower lumbar degeneration, more proximal lumbar levels may signify more extension. ${ }^{2}$ In such cases, the LDI will be lower while the magnitude of lordosis may remain relatively unaffected.

\section{RLL and HRQOL Scores}

Although PI - LL was developed based on HRQOL scores and RLL was developed based on mechanical complications, this study revealed that the correlation coefficient for the partial correlation with HRQOL scores at follow-up was higher for RLL than for PI - LL. This is reasonable because mechanical complications have been shown to affect HRQOL scores. ${ }^{28}$

\section{Limitations of the Study}

This study is a retrospective analysis of prospectively collected data. The ability to actually prevent mechanical complications by using RLL and LDI in preoperative planning has yet to be evaluated.

Because the calculations of RLL and LDI require multiplication and division, it is slightly more challenging to 
calculate them in one's head. However, once calculated, these values offer a significantly higher level of insight that makes it worth the effort. The use of automated tools should simplify the calculation.

\section{Conclusions}

As an absolute numeric value, PI - LL may be insufficient to quantify normolordosis for the whole spectrum of PI values in conjunction with previously reported thresholds. Furthermore, Schwab categories for PI - LL were found to constitute inhomogeneous groups of patients in this study, which may render them misleading when used for individualized surgical planning.

The PI-based proportional parameter of RLL offers an individualized quantification of LL for all PI sizes. RLL predicts mechanical complications more precisely and has better correlations with HRQOL scores than PI - LL.

The LDI quantifies the distribution of lordosis between the upper and lower arcs. Defining lordosis by using RLL and LDI together helps to interpret the patient's lordosis as the magnitude and distribution of lordosis in relation to his or her calculated ideals. The individualized understanding of the magnitude and distribution of lordosis offers a onesize-fits-all solution for the whole spectrum of PI.

The use of RLL and LDI together instead of PI - LL for surgical planning may result in better long-term HRQOL results with lower mechanical complication rates.

\section{References}

1. Asher MA, Min Lai S, Burton DC: Further development and validation of the Scoliosis Research Society (SRS) outcomes instrument. Spine (Phila Pa 1976) 25:2381-2386, 2000

2. Barrey C, Roussouly P, Le Huec JC, D’Acunzi G, Perrin G: Compensatory mechanisms contributing to keep the sagittal balance of the spine. Eur Spine J 22 (Suppl 6):S834-S841, 2013

3. Been E, Barash A, Marom A, Kramer PA: Vertebral bodies or discs: which contributes more to human-like lumbar lordosis? Clin Orthop Relat Res 468:1822-1829, 2010

4. Berge C: Heterochronic processes in human evolution: an ontogenetic analysis of the hominid pelvis. Am J Phys Anthropol 105:441-459, 1998

5. Bernhardt M, Bridwell KH: Segmental analysis of the sagittal plane alignment of the normal thoracic and lumbar spines and thoracolumbar junction. Spine (Phila Pa 1976) 14:717721, 1989

6. Berthonnaud E, Dimnet J, Roussouly P, Labelle H: Analysis of the sagittal balance of the spine and pelvis using shape and orientation parameters. J Spinal Disord Tech 18:40-47, 2005

7. Duval-Beaupère G, Schmidt C, Cosson P: A barycentremetric study of the sagittal shape of spine and pelvis: the conditions required for an economic standing position. Ann Biomed Eng 20:451-462, 1992

8. Fairbank JC, Couper J, Davies JB, O'Brien JP: The Oswestry low back pain disability questionnaire. Physiotherapy 66:271-273, 1980

9. Gelb DE, Lenke LG, Bridwell KH, Blanke K, McEnery KW: An analysis of sagittal spinal alignment in 100 asymptomatic middle and older aged volunteers. Spine (Phila Pa 1976) 20:1351-1358, 1995

10. Jackson RP, Kanemura T, Kawakami N, Hales C: Lumbopelvic lordosis and pelvic balance on repeated standing lateral radiographs of adult volunteers and untreated patients with constant low back pain. Spine (Phila Pa 1976) 25:575-586, 2000

11. Kuntz C IV, Levin LS, Ondra SL, Shaffrey CI, Morgan CJ: Neutral upright sagittal spinal alignment from the occiput to the pelvis in asymptomatic adults: a review and resynthesis of the literature. J Neurosurg Spine 6:104-112, 2007

12. Lafage V, Schwab F, Patel A, Hawkinson N, Farcy JP: Pelvic tilt and truncal inclination: two key radiographic parameters in the setting of adults with spinal deformity. Spine (Phila Pa 1976) 34:E599-E606, 2009

13. Le Huec JC, Aunoble S, Philippe L, Nicolas P: Pelvic parameters: origin and significance. Eur Spine J 20 (Suppl 5):564-571, 2011

14. Le Huec JC, Saddiki R, Franke J, Rigal J, Aunoble S: Equilibrium of the human body and the gravity line: the basics. Eur Spine J 20 (Suppl 5):558-563, 2011

15. Legaye J, Duval-Beaupère G, Hecquet J, Marty C: Pelvic incidence: a fundamental pelvic parameter for three-dimensional regulation of spinal sagittal curves. Eur Spine J 7:99-103, 1998

16. Mannion AF, Porchet F, Kleinstück FS, Lattig F, Jeszenszky $\mathrm{D}$, Bartanusz V: The quality of spine surgery from the patient's perspective. Part 1: The core outcome measures index in clinical practice. Eur Spine J 18 (Suppl 3):367-373, 2009

17. Nagelkerke NJD: A note on a general definition of the coefficient of determination. Biometrika 78:691-692, 1991

18. Obeid I, Hauger O, Aunoble S, Bourghli A, Pellet N, Vital JM: Global analysis of sagittal spinal alignment in major deformities: correlation between lack of lumbar lordosis and flexion of the knee. Eur Spine J 20 (Suppl 5):681-685, 2011

19. Polly DW Jr, Kilkelly FX, McHale KA, Asplund LM, Mulligan M, Chang AS: Measurement of lumbar lordosis. Evaluation of intraobserver, interobserver, and technique variability. Spine (Phila Pa 1976) 21:1530-1536, 1996

20. Roussouly P, Gollogly S, Berthonnaud E, Dimnet J: Classification of the normal variation in the sagittal alignment of the human lumbar spine and pelvis in the standing position. Spine (Phila Pa 1976) 30:346-353, 2005

21. Roussouly P, Gollogly S, Noseda O, Berthonnaud E, Dimnet $\mathrm{J}$ : The vertical projection of the sum of the ground reactive forces of a standing patient is not the same as the $\mathrm{C} 7$ plumb line: a radiographic study of the sagittal alignment of 153 asymptomatic volunteers. Spine (Phila Pa 1976) 31:E320E325, 2006

22. Roussouly P, Nnadi C: Sagittal plane deformity: an overview of interpretation and management. Eur Spine J 19:18241836,2010

23. Roussouly P, Pinheiro-Franco JL: Sagittal parameters of the spine: biomechanical approach. Eur Spine J 20 (Suppl 5):578-585, 2011

24. Schwab F, Patel A, Ungar B, Farcy JP, Lafage V: Adult spinal deformity-postoperative standing imbalance: how much can you tolerate? An overview of key parameters in assessing alignment and planning corrective surgery. Spine (Phila Pa 1976) 35:2224-2231, 2010

25. Schwab F, Ungar B, Blondel B, Buchowski J, Coe J, Deinlein $\mathrm{D}$, et al: Scoliosis Research Society-Schwab adult spinal deformity classification: a validation study. Spine (Phila Pa 1976) 37:1077-1082, 2012

26. Schwab FJ, Blondel B, Bess S, Hostin R, Shaffrey CI, Smith JS, et al: Radiographical spinopelvic parameters and disability in the setting of adult spinal deformity: a prospective multicenter analysis. Spine (Phila Pa 1976) 38:E803-E812, 2013

27. Schwab FJ, Diebo BG, Smith JS, Hostin RA, Shaffrey CI, Cunningham ME, et al: Fine-tuned surgical planning in adult spinal deformity: determining the lumbar lordosis necessary by accounting for both thoracic kyphosis and pelvic incidence. Spine J 14 (11 Suppl):S73, 2014 
28. Soroceanu A, Diebo BG, Burton D, Smith JS, Deviren V, Shaffrey C, et al: Radiographical and implant-related complications in adult spinal deformity surgery: incidence, patient risk factors, and impact on health-related quality of life. Spine (Phila Pa 1976) 40:1414-1421, 2015

29. Sparrey CJ, Bailey JF, Safaee M, Clark AJ, Lafage V, Schwab F, et al: Etiology of lumbar lordosis and its pathophysiology: a review of the evolution of lumbar lordosis, and the mechanics and biology of lumbar degeneration. Neurosurg Focus 36(5):E1, 2014

30. Stagnara P, De Mauroy JC, Dran G, Gonon GP, Costanzo G, Dimnet J, et al: Reciprocal angulation of vertebral bodies in a sagittal plane: approach to references for the evaluation of kyphosis and lordosis. Spine (Phila Pa 1976) 7:335-342, 1982

31. Vedantam R, Lenke LG, Keeney JA, Bridwell KH: Comparison of standing sagittal spinal alignment in asymptomatic adolescents and adults. Spine (Phila Pa 1976) 23:211-215, 1998

32. Vialle R, Levassor N, Rillardon L, Templier A, Skalli W, Guigui P: Radiographic analysis of the sagittal alignment and balance of the spine in asymptomatic subjects. J Bone Joint Surg Am 87:260-267, 2005

33. Ware JE Jr, Sherbourne CD: The MOS 36-item short-form health survey (SF-36). I. Conceptual framework and item selection. Med Care 30:473-483, 1992

34. Yilgor C, Sogunmez N, Boissiere L, Yavuz Y, Obeid I, Kleinstück F, et al: Global Alignment and Proportion (GAP) Score: development and validation of a new method of analyzing spinopelvic alignment to predict mechanical complications after adult spinal deformity surgery. J Bone Joint Surg Am 99:1661-1672, 2017

\section{Disclosures}

Professor Acaroğlu has received clinical or research support for the study described (includes equipment or material) from DePuy Synthes and has received support of non-study-related clinical or research efforts from Medtronic; he has also received grants/ research support from Stryker Spine; he is on the Speaker's Bureau for AO Spine, Medtronic, Stryker Spine, and Zimmer Biomet; he is on the Advisory Board/Panel for AO Spine; and he is a stock/shareholder of incredX (self-managed). Dr. Alanay has received grants/research support from DePuy Synthes and is a consultant for DePuy Synthes, Stryker Spine, and Medtronic. Dr. Boissière is a consultant for Spineart and has received clinical or research support for the study described (includes equipment or material) from DePuy. Dr. Kleinstück has received clinical or research support for the study described (includes equipment or material) from DePuy Synthes and is a member of the Speaker's Bureau for DePuy Synthes. Mr. Obeid is a consultant for DePuy Synthes and Medtronic; he has received clinical or research support for the study described (includes equipment or material) from DePuy Synthes; he receives royalties from Clariance, Alphatec, and Spineart. Professor Pellise is a consultant for DePuy Synthes Spine, Medtronic, and Zimmer Biomet; he has received support of non-study-related clinical or research efforts from DePuy Synthes Spine, Medtronic, and Zimmer Biomet; he has also received grants/research support from K2M. Dr. Pérez-Grueso is a consultant for DePuy Synthes and K2M; he has received grant/research support from DePuy Synthes. Mrs. Sogunmez has received grants/research support from DePuy Synthes. The European Spine Study Group has received grants/research support from DePuy Synthes.

\section{Author Contributions}

Conception and design: Alanay, Yilgor, Yavuz. Acquisition of data: all authors. Analysis and interpretation of data: Alanay, Yilgor, Sogunmez, Yavuz, Abul, Boissière, Haddad. Drafting the article: Alanay, Yilgor, Yavuz. Critically revising the article: Mannion. Reviewed submitted version of manuscript: all authors. Statistical analysis: Alanay, Yilgor, Yavuz, Abul. Study supervision: Alanay.

\section{Correspondence}

Ahmet Alanay, Comprehensive Spine Center at Acibadem Maslak Hospital, Buyukdere Cad No: 40, 34457 Maslak, Sariyer, Istanbul 34100, Turkey. email: aalanay@gmail.com. 